\title{
Natural Interaction Design for Navigation in Virtual Environment of Sino-Portuguese Architecture in Museum
}

\author{
Kosin KALARAT* and Sasithorn RATTANARUNGROT \\ Division of Multimedia Technology and Animation, School of Informatics, Walailak University, \\ Nakhon Si Thammarat 80160, Thailand
}

('Corresponding author's e-mail: kosin.ka@wu.ac.th)

Received: 4 March 2019, Revised: 6 November 2019, Accepted: 8 December 2019

\begin{abstract}
Virtual Environment (VE) is an artificial environment created for providing the information in Virtual Reality (VR). The audiences are able to obtain the information via interaction to the environment. Virtual Reality for Culture heritage buildings, which are inherited from past generations and maintained in the present, is an interested topic because its contents often built for giving the information and knowledge about the past to the new generation in virtual museums. However, there is a limitation of Virtual Reality (VR) applications in reproducing cultural sites as most of these applications are not successful in catching public's attention and involvement. In this research, we focus on using Natural Interaction (NI) interfaces based on a body movement in Virtual Reality for museums to grab attention from audience and to increase public's involvement. This paper presents the development of Natural Interaction using Microsoft Kinect to define the new grammar of gesture for Sino Portuguese Architecture which is a cultural heritage of Thailand. Results of early tests improved the prototype in progress which alllows more complexity in natural interchanges and connection between users and virtual world. The final result was evaluated after the system was installed in Peranakannitat museum and by interviewing 6 docents. It was found that our system was easy to use as it took only about $3-5$ min to familiarize the system for navigation in the virtual environment without recognizing the grammar of gesture commands.
\end{abstract}

Keywords: Virtual Reality, Virtual Environment, Virtual Museum, Natural Interaction, Culture Heritage

\section{Introduction}

One of the institutions that concerns about collecting unique objects and forms the core of its activities for exhibitions, education and research, is a museum. The objects collected may be artistic, cultural, historical, or scientific which are able to transfer a historical information to the audience. Many museums establish permanent or temporary exhibitions to provide information and to educate the public audience. Additionally, a museum can also be an entertainment place for families especially for children to enjoy at the same time learn.

In the digital era, digital technology has come to play a role in providing the information to the museums. The digital data is applied to every part in the museum such as light, sound and visual system to augment the museum's ability to communicate or educate. Most traditional museums collect objects and display the history through text or by an explanation by a tour guide. In contrast, modern museums, which are enhanced by the digital technology, combine the high performance capacity of storage in digital data and the high speed computational power to create contents in multimedia such as text, sound, video, animation, Augmented Reality (AR), Virtual Reality (VR) and interaction system to obtain the whole information without any aid for explaination. 
http://wjst.wu.ac.th

A virtual museum is one of the museums that use digital technology by applying the information to the audience in order to augment, enhance, or complement the experience through personalization in the museum, interactivity and various contents. Particularly, the content that involves archaeological site, it is not necessary for visitors anymore to go to the real place and obtain an experience for the site, as they are able to access the location and interact with the system using virtual space.

Therefore, Virtual Reality (VR) is applied to the museum to augment the traditional museum. VR is able to provide experience to users by simulating the situation, environment, atmosphere or objects through virtual world. Virtual Reality (VR) is a medium used to simulate 3D environment using computer technology for interaction which leads user feel immersed to the environment. The environment in VR, which is called Virtual Environment (VE) or Virtual World, is the content of a given medium. VR has been applied widely such as education, healthcare, military, game and cultural heritage. The user will receive the information, experience, and atmosphere of the virtual world which is the $3 \mathrm{D}$ environment in VR. Moreover, the user is able to interact to the VE element.

This research selected one of the Sino Portuguese architectures that is called "Shop-house" which is a 2-storey shop house built for a living. The system allows the user to explore inside the house which simulates lifestyle in the past and provide the information via storytelling for each part of the house. Natural Interaction using Microsoft Kinect is the main device to track user's movement to control user's perspective and the direction to walkthrough inside Virtual Environment. Finally, this research observed the user who interacted to our system to evaluate the gesture design if it is suitable for navigation or not,.

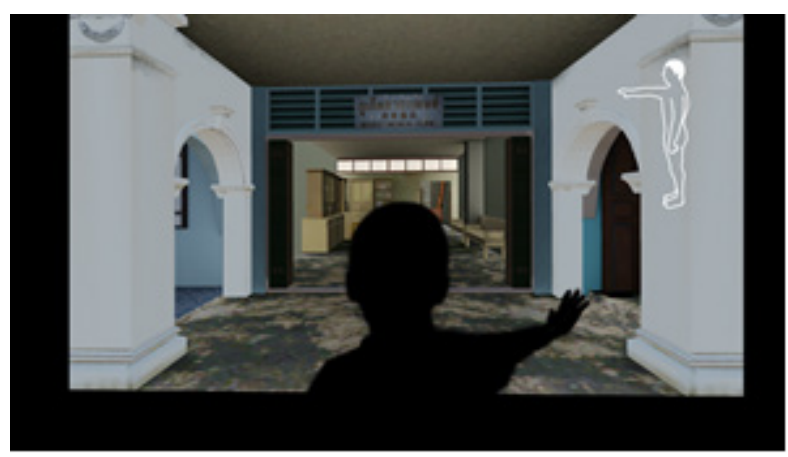

Figure 1 The user can learn the gestures in order to move in the Virtual Environment.

\section{Background}

Sino Portuguese Architecture

Sino Portuguese Architecture was built around A.D.1890 - 1920 in Phuket, Thailand. The architecture is a combination of East and West building styles, making it one of the unique architecture heritages in the country. The architecture is interesting as it is influenced by many building styles from Siamese, Chinese, Malaysian to European [1]. The Sino Portuguese decorative facade is influenced by the European as evident in the form of plant emphasizing interflow and spiral vines, leaves and flower [2]. However, the doors and windows are Chinese style. Generally, there are many types of Sino-Portuguese Architecture building such as Shop-house, mansion, government building, etc.

This research selects the Shop-house to be an environment for our Virtual Reality research because it is a 2-storey house and it has a long shape as showed in Figure 2. To determine the grammar of Natural Interaction [3], the user has to explore from ground floor to the 2nd floor. Likewise, another reason for choosing the place is that the building represents the unique Sino-Portuguese design, as there is a hole on the roof or air well at the middle of house to ventilate and get sunshine or moonshine. Besides, there is a well to gather water for consumption. 
http://wjst.wu.ac.th

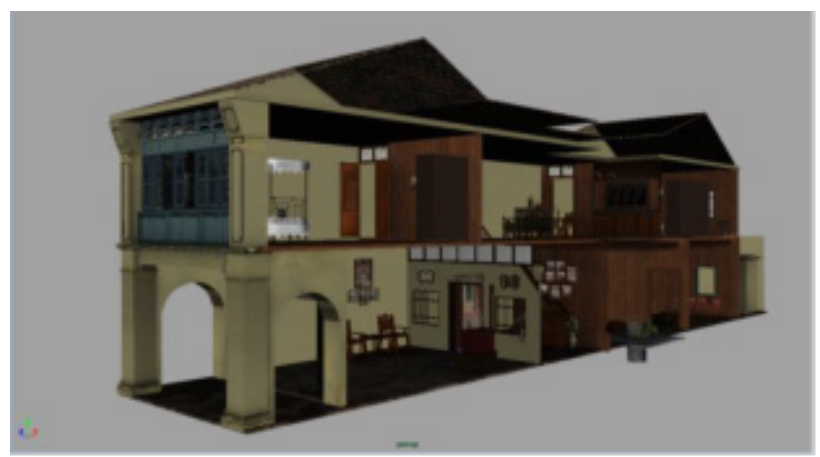

Figure 2 Shows the interior inside Shop-house.

\section{Virtual Reality Walkthrough}

Virtual Reality Walkthrough is a way to present information in Virtual Reality. The users can survey around the virtual environment [4] like they are walking in the real world. The VR walkthrough normally is used to represent constructions or architectures that users could get information to learn the history [5], art and tradition simultaneously. This paper uses game engine, Unity3D, to be a platform to implement the virtual environment and support real-time interaction. Semi-immersive Virtual Reality is applied for this research using Natural Interaction Interface to control user perspective and explore inside the virtual world in real-time.

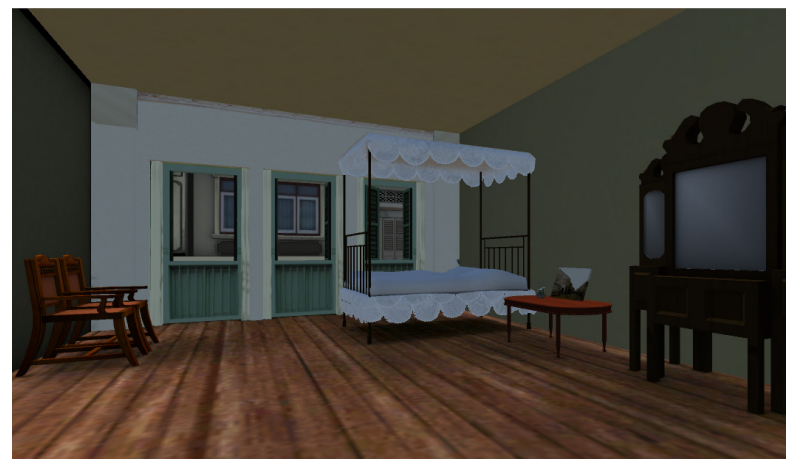

Figure 3 User can explore in virtual space by walkthrough in Virtual Reality.

\section{D modeling}

For 3D Virtual Environment establishment, we started by collecting the information of Shop-house from Phuket official document [1] for the houses' structure and old pictures from Thai-Hua museum. The software that we used for creating the 3D model was Autodesk Maya. In other words, we only included house and the objects that were in it such as lamps, wells and beds, etc. The application includes 5 different houses with the antique properties and imports the asset to Unity3D scene. 
http://wjst.wu.ac.th

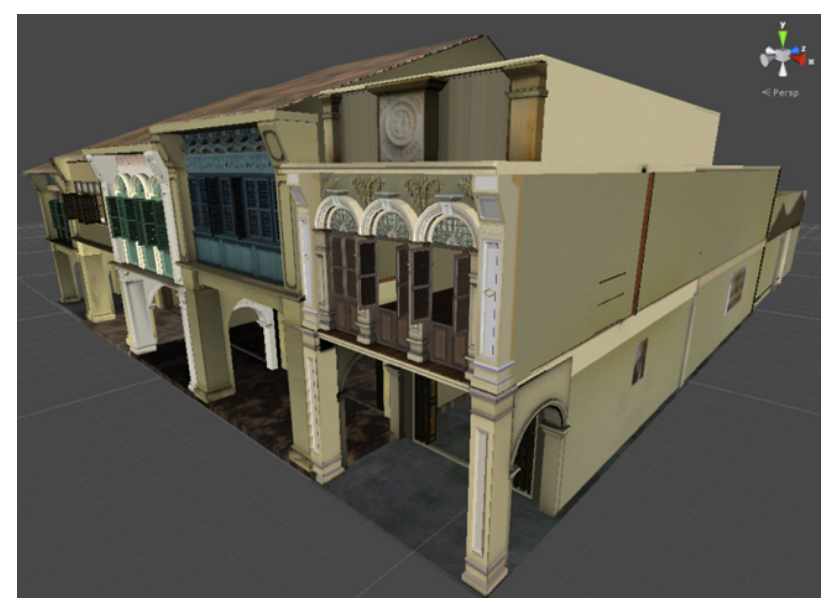

Figure 4 Virtual building of Shop-house.

\section{Natural Interaction}

Natural Interaction (NI) means an interaction in the natural way to communicate with computer or system. Gesture-based interaction, one of Natural Interaction, is a new paradigm in Human-Computer Interaction (HCI) which is able to control a technical system with gesture or users will no longer need to use any extra device [6] such as joystick, mouse and keyboard. The users can only use their body or hand to control the system via their own gesture. Gesture-based interaction widely used for 2 application domains for research which are Virtual Reality and Augmented Reality.

This research applied the application about VR applying Natural Interaction [7] to cultural heritage applications by using Microsoft Kinect. Microsoft Kinect is a low-cost device for tracking human's gesture and sensing the input devices particularly created for game purpose. However, it has been applied for this research to control exploration system by users' gesture in Virtual Environment that simulate heritage site used for the museum.

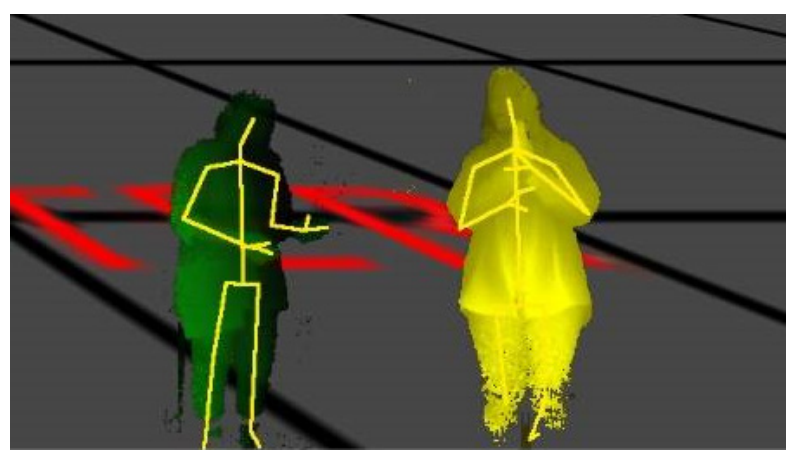

Figure 5 Skeleton tracking by Microsoft Kinect for Natural Interaction. 
http://wjst.wu.ac.th

\section{Research approach}

To explore around the Shop-house which was set back in the past for our Virtual Reality application in Peranakannitat museum, we identified some limitations.

First is the limitation of the involvement [8] of the visitors who come in the museum. Most visitors should be able to participate with Virtual Reality system [9].

Second is the limitation of the space in the museum. The museum allowed the available area for installation and having activity about $1.2 \mathrm{~m}^{2}$ with a wall. The area is a bit small for setting up a fullyimmersive Virtual Reality.

For the final limitation, we were concerned about the maintenance cost after the system would be available for the visitors.

With the concerned restrictions, we tried to find the solutions that fit for all limitations. Thus, we separated each limitation and found out that the possibility system is the most suitable in system design process.

\section{Semi - Immersion Virtual Reality}

There are many kinds of Virtual Reality divided by display device, immersive level, etc. For the immersive level of VR, we intend to use the fully-immersive VR [10] because visitors would be able to receive all of information provided in Virtual Environment. Head Mouse Display (HMD) and Cave Automatic Virtual Environment (CAVE) are 2 choices considered by researcher. For HMD, this kind of devices allows a user to see all around Virtual Environment via stereoscopic headset. However, it was resisted by the fist first and the 3rd limitation on the involvement and maintenance cost. HMD normally provides personal information to a person; however, it lacks involvement. Because visitors have to wear a headset device for accessing the artificial environment, there is a maintenance cost that has to be considered. Therefore, we move to CAVE decision. VR in CAVE is a good choice to solve the involvement problem because the visitors, who are outside the CAVE, are able to see following the accessing visitor's perspective when he/she explore virtual space. Nevertheless, CAVE still stuck with the 2nd limitation because CAVE has to take quite big place for installation. Because of that, we reduced the immersive level to Semi-Immersive level [11] to cover all requirements.

For Semi-Immersive VR, we selected a desktop VR because this system would not spend much installation space. It used only 1 wall to suspend a big television screen for displaying visitor's perspective exploration in real-time. Visitors are able to immerse into the virtual world while realizing that they are still in the real world.

\section{Natural interaction interface}

After we have selected the immersive level to come across with the $1^{\text {st }}$ and $2^{\text {nd }}$ limitation, the last limitation would be considered by an appropriated interaction. We have to consider which interactions which does not require visitors to contact with the input devices directly in order to reducing device deterioration.

Natural Interaction Interface is an interaction based on only visitors' body movement [12] without required any touched devices. This research used Microsoft Kinect which is a mark-less sensor. Microsoft Kinect is mainly built for video game industry to track the users' body that is playing a game. It is an opportunity to apply this device to this project for capturing visitors' gesture, called gesture-based interaction, to control in Virtual Environment of Cultural Heritage exploration.

\section{Gesture design}

After we decided to use gesture-based interaction [13], we designed how to control the exploration in VE by gesture in the natural way. This means that a visitor could survey inside the virtual 3D environment using his/her own body gesturing [14]. Moreover, visitors around the user are able to participate with the virtual environment through the big screen TV even if they do not control by themselves. 
http://wjst.wu.ac.th

First of all, we defined how to move in virtual 3D space. Because of the limitation of space, we minimized the usage area that is able to receive the command from 1 user. The system has to be specified the position, called hotspot, on the floor in order to limit user repositioning when they access to the system. There are 2 hotspots to control the virtual camera moving and stopping which are showed in picture below.

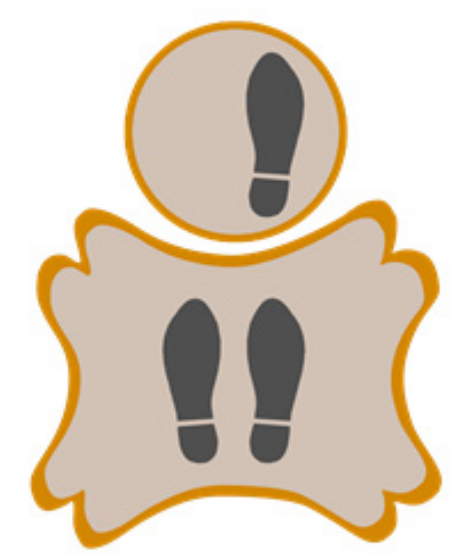

Figure 6 The 2 Hotspots on the floor.

Figure 6 shows the hotspots on the floor to guide user how to walkthrough in virtual space. The 1 st hotspot includes a sign of 2 feet which means unchanged position in the virtual world. The 2 nd hotspot is a sign that consists of only 1 foot which means move forward in the virtual world. A user can decide whether move forward or stop by stand on the hotspots. For the moving forward, a user can use only right lag step into the circle hotspot to make participant feel moving forward.

Secondly, we designed how users change their perspective view and change view's direction when they are moving. For perspective controlling, we decide to use only right hand to manage the screen's view in the real world and to control a camera in the virtual world. Using right hand, users will be less confused than using another hand while they are using right foot at the same time. That means the users are able to change their perspective while they are moving. When the participants changed their view during the movement, they can also change direction of their own moving because the system was set for moving only forward way as showed in Figure 7. 


\section{Manual}

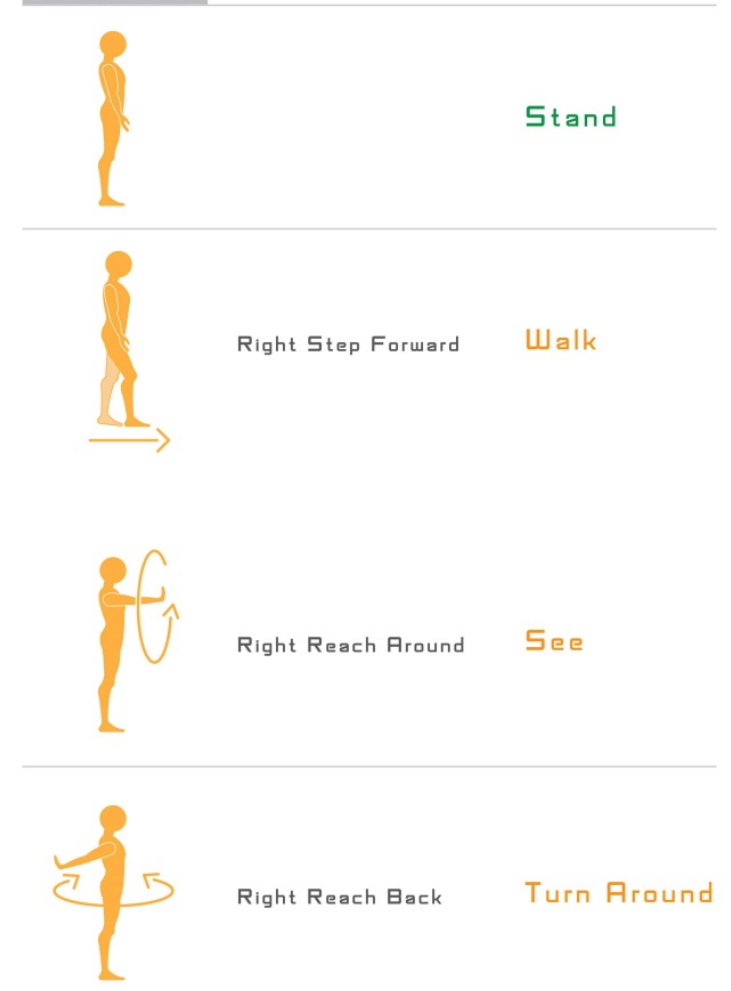

Figure 7 Interaction manual for visitors.

Figure 7 shows the manual of this VR interaction which uses only right hand and right foot to reduce the usage confusion. In addition to turn around, the users have to change their perspective over 180 degrees in horizontal by extending the arm and keeping the arm behind the chest as showed in Figure 8.
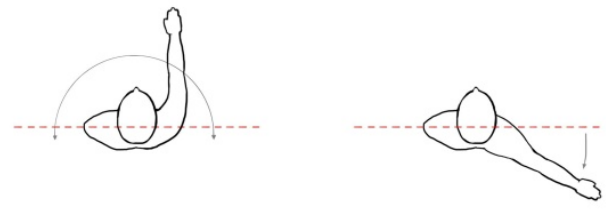

Figure 8 Shows the top view of activated user to turn around camera in the virtual world. 


\section{Virtual environment exploration}

These solutions try to make a natural interaction which is friendly for every age to enjoy the content and perceive the history simultaneously. This system used 65 " TV to display the exploration result from the user's gesture producing a sensation of immersion. While the active user is controlling the system, other visitors also can learn the information through visualizing and listening to the cultural contents in a passive way. Thus there is always an opportunity to engage inactive exploration.

The application is implemented as a Culture Heritage with Virtual Reality in Unity3D, Game Engine, using Microsoft Kinect as a sensor to capture the user's gesture. To be effective body tracking, Kinect had to be installed for full body capturing. Due to the limited dimensions of the room, the Kinect had to be high from the floor about $1.2 \mathrm{~m}$ below the screen, and $1.2 \mathrm{~m}$ distance from the standing hotspot to capture whole body of activated visitor.

For this research, the application has to calibrate and measure the user's skeleton in order to be an input when user starts gesturing. The system only allows 1 user to activate the VR exploration by standing on the hotspot. The system will ignore other users who stand outside the hotspot even though they come into the system area.

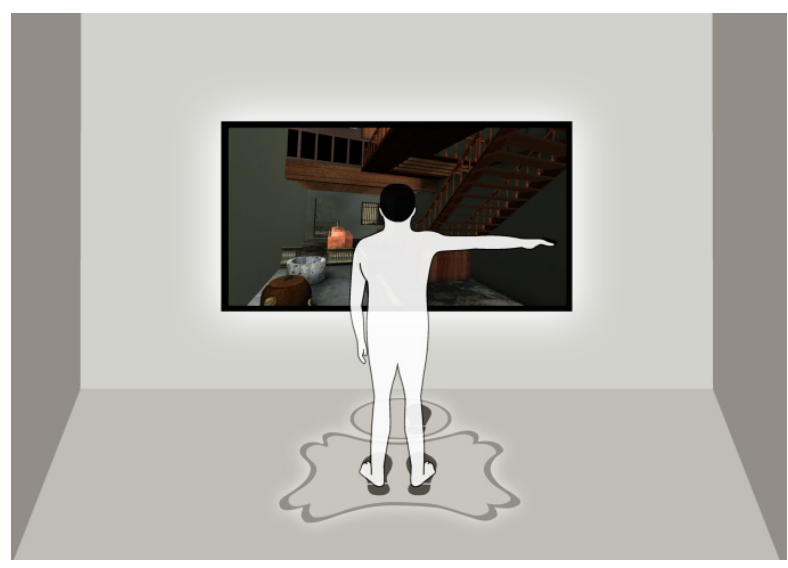

Figure 9 The system includes hotspots, a monitor screen and a sensor (Kinect).

Figure 9 shows the overall system that includes a big screen monitor to display user's perspective in real-time, 2 hotspots to fix the user's position, and Microsoft Kinect sensor to track the movement of user.

\section{Results and discussion}

There are 3 observational approaches for this research. The 1st observational approach was determined through some technical issues concerning the limitation of the museum. We created the mockup space and system as same as the museum requirement and adjusted our system such as display, sound and interaction system. Then, we examined the user on the interaction regarding usability, length, naturalness and safety of interaction.

The result of the observation and evaluation in this stage were informed to programmers and artist to fix both program and content error. For the program issue, one of the big issues was the velocity of camera movement when the user turns or turns around in the Shop-house. It made the user felt dizzy from the sudden turns while moving forward. Because of that, we decreased the rotation speed of camera when the user was exploring in the virtual world. 
http://wjst.wu.ac.th

For the 2nd approach, we had an opportunity to temporarily install our system in a local department store. The 2nd evaluation stage combined with observation and interview by the visitors. The observations allowed us to continue evaluating the usability, time to learn, and subjective satisfaction.

In the interviews section, the system was tested by 30 participants randomly. We received many feedbacks from the visitors such as subjective opinions, usability, and interactivity, level of immersion approach, the learning content and satisfaction of system.

After collecting the observation feedback, it was found that the system was easy to use for all ages, from school children to senior. They took not much time to familiarize [15] the system with interaction. The average time to get into the system was about 2 - 3 min and they normally spent time exploring in the Shop-house for about 7 - $10 \mathrm{~min}$.

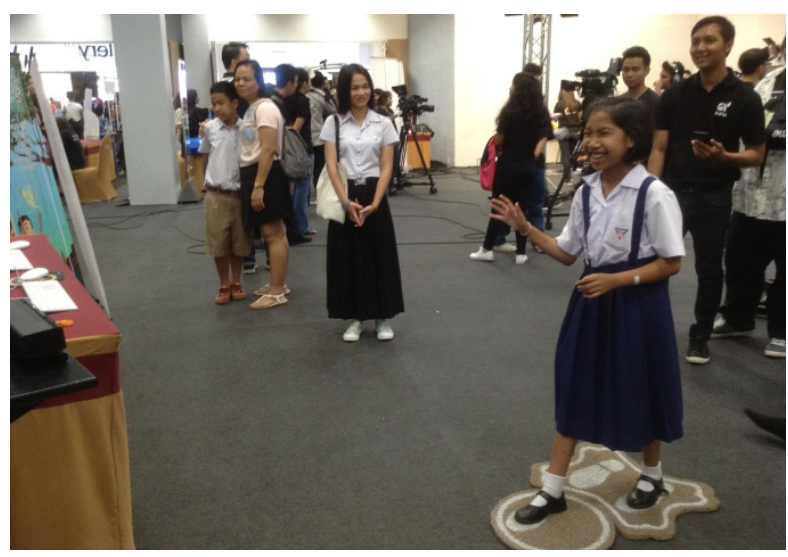

Figure 10 Shows the mock-up hotspot on the floor.

For the interviews feedback, the result reported the visitors' opinion after they got the experience from our system. The results showed that people were impressed about exploring around the Shop-house and seeing the antique objects which were decorated inside the house. Most people responded that they felt convenient to use our Natural Interaction because they had no need to recognize the grammar from gesture design. They were able to survey the Virtual Environment instinctively. Some visitors' commented that our system allowed them to have an enjoyable exploration making them involved even though they did not control the system by themselves.

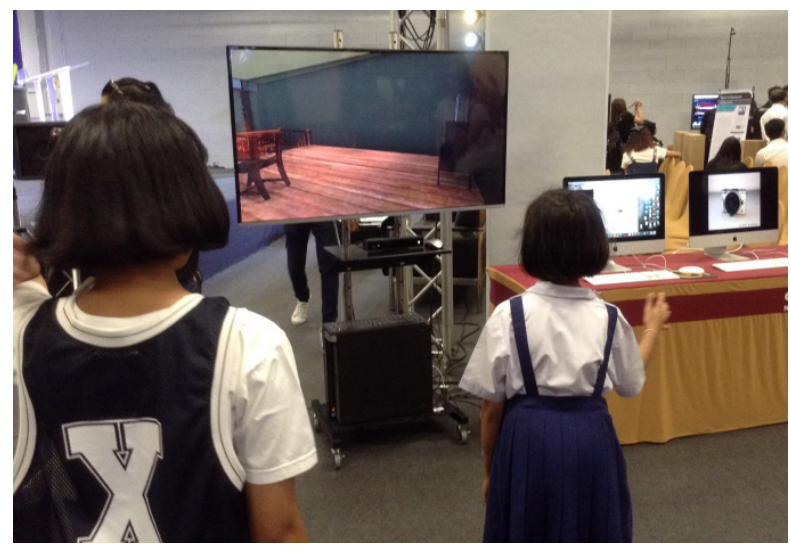

Figure 11 Visitors can involve the VR system even if they do not access by themselves. 
http://wjst.wu.ac.th

However, there were some comments from users suggesting improvement of our system such as the volume of sound system, reflect on the monitor screen interference, some visitors move pass in front of the activated visitor that make the sensor restricted, etc. Every comment from the users was collected by the researchers for the improvement of the system.

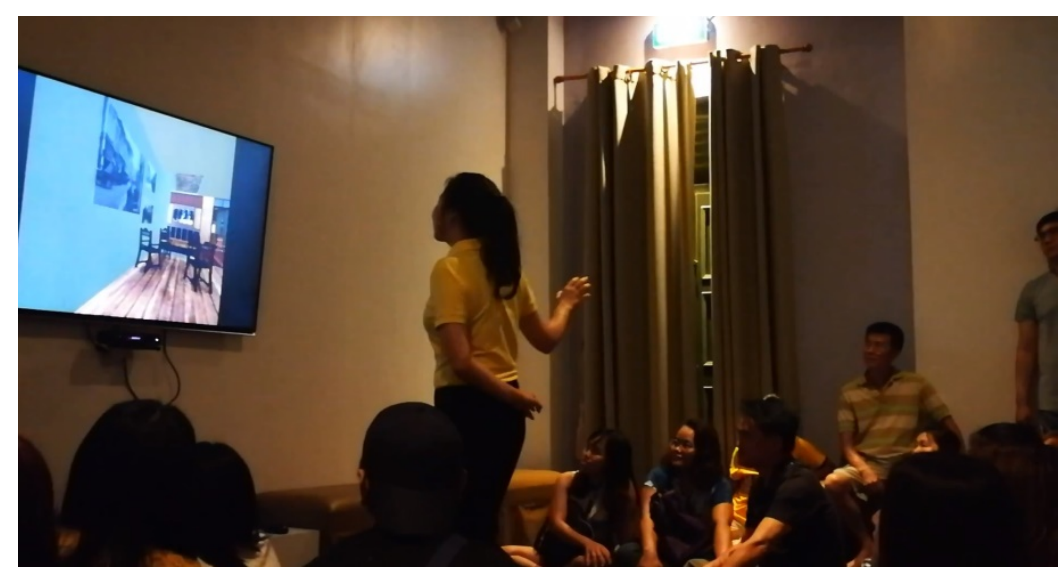

Figure 12 A docent for the museum illustrate the virtual Shop-house through the visitors.

For the final approach, we observed and interviewed the users in the museum after the system was installed on the site. However, there was a change in the procedure as visitors were not allowed to visit each section in the museum by themselves. The visitors were separated in a group with a round per hour. Another thing was, there were docents in the museum who provided the visitors the guide to reach every museum's section and give information to the audiences as shown in Figure 12. Thus, we have to change our system by muting our narrative sound because the docent illustrated the content by themselves. Moreover, the target group also changed from only visitors to, docents and visitors, to evaluate our interaction system.

After we evaluate the result by interviewing 6 docents, the interviews provided insight into the docents' opinions of experiencing on navigating in Virtual Sino-Portuguese Architecture. The results showed us that all docents commented that the interaction is easy to use as they spent time training and familiarizing the navigation task about $3-5 \mathrm{~min}$ and it was not necessary to recognize the grammar of gesture commands. Another thing is, our interactive system was impressive to the visitors as they are curious about how to control the system and this gives them more attention to the content of the building. Moreover, after this session was finished, some visitors have tried to control the system by themselves enjoyably.

\section{Conclusions}

In this paper, we introduce the new way to navigate the Virtual Environment with Natural Interaction applying for virtual building called Shop-house which is Sino-Portuguese Architecture. The system has been embedded in 1 section of Peranakannitat museum at Phuket, Thailand.

By using the Natural Interaction Interface to explore the building, the visitors felt interested on the system as people, who come in the museum, are wondering how the exploration of the place could be controlled by human's body or gesture. Visitors are able to get involved with the system by viewing the screen when another visitor is exploring the house. Moreover, some visitors have been waiting for another user to finish his/her exploration and try to control by themselves. That means not only the system makes, the visitors to get to learn the history, but also it makes the people enjoyed when they are gesturing. However, the system has to be developed more features such as selection feature as it would have many 
http://wjst.wu.ac.th

kinds of building in Sino-Portuguese Architecture to represent every style of houses. Therefore, docents must be able to select other kind of buildings for navigation and to illustrate the character of each house in the future.

\section{Acknowledgements}

This work was partly supported by Multimedia Technology and Animation, School of Informatics, Walailak University, Thailand. We would like to thank Phuket municipality for providing the information of the Sino-Portuguese architecture and Kazar Media Co., LTD for providing an opportunity to cooperate to the part of virtual museum in Peranakannitat museum, Phuket.

\section{References}

[1] Official Fine Art Department. The Explore of Phuket History, 1987.

[2] P Tepsing and W Watanasin. 2004, Decorative ornament on facade of Sino-Portuguese building in Phuket province from Department of Educational Foundation. Master Thesis, Faculty of Liberal Art, Prince of Songkla University, Thailand.

[3] E Pietroni, A Pagano and C Rufa. The etruscanning project: Gesture-based interaction and user experience in the virtual reconstruction of the regolini-galassi tomb. In: Proceedings of the IEEE Digital Heritage International Congress. 2013, p. 653-60.

[4] J Zara. Virtual Reality and Cultural Heritage on the Web. In: Proceedings of the $7^{\text {th }}$ International Conference on Computer Graphics and Artificial Intelligence. 2004, p. 101-12.

[5] P Aiello, F D'Elia, SD Tore and M Sibilio. A constructivist approach to virtual reality for experiential learning. E-Learn. Digit. Media 2012; 9, 317-24.

[6] L Baraldi, F Paci, G Serra, L Benini and R Cucchiara. Gesture recognition using wearable vision sensors to enhance visitors' museum experiences. IEEE Sens. J. 2015; 15, 2705-14.

[7] T Alisi, A Del Bimbo and A Valli. Natural interfaces to enhance visitors' experiences. In: Proceedings of the IEEE Multimedia. 2005, p. 80-5.

[8] ML Rayan. Narrative as Virtual Reality: Immersion and interactivity in literature and electronic media for Baltimore and London. Hopkins University Press, HB, 2001, p. 259-62.

[9] E Pietroni, C Ray, C Rufa, D Pletinckx and I Van Kampen. Natural interaction in VR environments for cultural heritage and its impact inside museums: The etruscanning project. In: Proceedings of the IEEE $18^{\text {th }}$ International Conference on Virtual Systems and Multimedia. 2012, p. 339-46.

[10] M Carrozzino and M Bergamasco. Beyond virtual museums: Experiencing immersive virtual reality in real museums. J. Cult. Herit. 2010; 11, 452-8.

[11] B Leibe, T Starner, W Ribarsky, Z Wartell, D Krum, B Singletary and L Hodges. The perceptive workbench: Toward spontaneous and natural interaction in semi-immersive virtual environments. In: Proceedings of IEEE Virtual Reality 2000. 2000, p. 13-20.

[12] G Castellano, R Bresin, A Camurri and G Volpe. Expressive control of music and visual media by full-body movement. In: Proceedings of the Conference on New Interfaces for Musical Expression 2007. 2007, p. 390-1.

[13] L Rodrigues da Silva, LS Garcia and L Silva. Gesture vocabulary for natural interaction with virtual museums. In: Proceedings of the $16^{\text {th }}$ International Conference on Enterprise Information Systems. 2014, p. 5-13.

[14] G Castellano, SD Villalba and A Camurri. Recognizing human emotions from body movement and gesture dynamics. In: Proceedings of the $2^{\text {nd }}$ International Conference on Affective Computing and Intelligent Interaction. Lisbon, 2007.

[15] E Pietroni. Natural interaction in VR environments for Cultural Heritage: The virtual reconstruction of the Regolini Galassi Tomb in Cerveteri. Archeol. Calc. 2013; 24, 231-47. 